\title{
RECONHECIMENTO DE EXEMPLARES DE HOVENIA DULCIS THUNBERG, NA ARBORIZAÇÃO URBANA DE CURITIBA - PR Helayne Aparecida Maieves ${ }^{12}$ e Rosemary Hoffmann Ribani $^{3}$
}

\section{RESUMO}

Hovenia dulcis Thunberg, conhecida vulgarmente por uva-do-japão (PR), pertence à família Rhamnaceae e ocorre naturalmente na China, Japão e Coréias. A espécie vem sendo cultivada na Argentina, Paraguai e no sul do Brasil, principalmente nas regiões de climas Cfa, Cfb e Cwa, segundo a classificação de Köppen e, de forma isolada ou em pequenos povoamentos. O fruto é uma pequena cápsula globosa seca de $6 \mathrm{~mm}$ a $7 \mathrm{~mm}$ de diâmetro, contendo 2 a 4 sementes, preso ao pedúnculo cor de canela, que se torna espessado e carnoso ao madurar, com sabor doce e agradável. Este trabalho teve como objetivo a identificação dos exemplares de Hovenia dulcis Thunberg, encontradas na arborização de ruas, parques e praças, escolas, faculdades e universidades além de residências na cidade de Curitiba - PR. Foram encontrados ao todo 32 exemplares de Hovenia dulcis Thunberg, nos 9 bairros pesquisados (Jardim das Américas, Santo Inácio, Centro, Alto da XV, Alto da Glória, Cristo Rei, Jardim Botânico, Guabirotuba e Hauer). Considerada como espécie invasora, a Secretaria de Municipal do Meio Ambiente do município, retira uma espécie exótica invasora, para plantar cinco árvores nativas. Apesar de serem consideradas invasoras, por não ser nativa da área em estudo, a uva-do-japão, apresenta uma madeira apreciável, apresenta efeitos alelopáticos em sementes de alface, suas folhas possuem substâncias como as saponinas triterpênicas, muito estudadas na área de farmacologia, por possuírem atividade antiinflamatória, seus frutos são doces quando maduros e são alvo de estudos sobre seus teores de substâncias anticancerígenas, não é indicada para alimentação animal, por desencadear a doença Polioencefalomalacia (PEM). Espécies frutíferas em arborização urbana é alvo de vandalismo, além de serem consideradas disseminadoras de doenças, por servirem de alimento para vetores, tais como moscas, ratos e barata e sujarem os pavimentos.

Palavras-chave: Uva-do-japão; Espécie invasora; Melia azedarach.

\section{IDENTIFICATION OF SPECIMENS OF HOVENIA DULCIS THUNBERG, IN URBAN ARBORIZATION THE CURITIBA - PR}

\begin{abstract}
This study aimed to identify trees copies, of Hovenia dulcis Thunberg, found in the greening of streets, parks and plazas, schools, colleges and universities in addition to residences in the city of Curitiba - PR. There were found at all 32 specimens of Hovenia dulcis Thunberg, in nine districts surveyed (Jardim das Américas, Santo Inácio, Centro, Alto da XV, Alto da Glória, Cristo Rei, Jardim Botânico, Guabirotuba and Hauer). Considered an invasive species, the Secretary of the Municipal Environment of the city, takes an invasive alien species, five native trees to plant. Although they are considered invasive because it is not native to the area under study, the japanese grape, presents a considerable wood, has allelopathic effects on lettuce seed, its leaves are substances such as triterpene saponins, much studied in the area of pharmacology, because they have anti-inflammatory activity, its fruits are sweet when ripe and are the focus of studies on their levels of anti-cancer substances, is not suitable for animal feed, by triggering the disease Polioencephalomalacia (PEM). Fruit species in urban forestry is vandalized, and are considered as disseminators of disease, for serving as food for vectors such as flies, rats and roachs and to dirty pavements.
\end{abstract}

Keys-word: Japanese grape; Invasive species; Melia azedarach.

\footnotetext{
1 recebido em 28.04.2012 e aceito para publicação em 15.03.2013

2 Doutoranda em Tecnologia de Alimentos - Universidade Federal do Paraná.

3 Docente Engenharia Química e Engenharia de Alimentos - Universidade Federal do Paraná. E-mail: helaynemaieves@agronoma.eng.br.
} 


\section{INTRODUÇÃO}

Hovenia dulcis Thunberg, conhecida vulgarmente por uva-do-japão (PR), banana-do- japão (SC), caju-do-japão (RJ), mata-fome (SP), pau-doce (MG) e chico-magro (SP), pertence à família Rhamnaceae e ocorre naturalmente entre $25^{\circ}$ e $41^{\circ}$ $\mathrm{N}$ e $100^{\circ}$ e $142^{\circ} \mathrm{L}$ leste, principalmente, na China, Japão e Coréias. (RIGATTO et al., 2001).

Para Carvalho (1994) quanto à etimologia, o nome dado ao gênero "Hovenia" foi em homenagem a David Hoven (1724-1787), senador de Amsterdã, que ajudou Thunberg a financiar sua expedição ao sul da África, Java e Japão. Já o nome da espécie “dulcis” vem do latim dulcis, que significa doçura dos eixos das infrutescências.

Segundo Rigatto e colaboradores (2001), a espécie vem sendo cultivada na Argentina, Paraguai e no sul do Brasil, principalmente nas regiões de climas Cfa, Cfb e Cwa, segundo a classificação de Köppen e, de forma isolada ou em pequenos povoamentos. Em nosso país, a bacia do Rio Uruguai, nos estados de Santa Catarina e Rio Grande do Sul, no sudoeste do Paraná, são comuns pequenos plantios de uvado-japão, em propriedades agrícolas, implantados por mudas ou, eventualmente, por semeadura direta no terreno.

É uma planta heliófila, caducifólia, alcançando alturas de 10 a $15 \mathrm{~m}$, podendo chegar até a $25 \mathrm{~m}$, com copa globosa e ampla. Seu diâmetro médio à altura do peito varia entre 20 e $40 \mathrm{~cm}$ podendo chegar a $50 \mathrm{~cm}$. Seu tronco geralmente é reto e cilíndrico, apresentando fuste com até $8 \mathrm{~m}$ de comprimento, ramificação dicotômica, com ramos pubescentes enquanto jovem. Apresenta gemas dormentes subcorticais, rebrotando intensamente da touça, podendo ser manejada por talhadia, com rotações previstas de 10 a 15 anos (SELLE, 2009).

Quanto à sua fenologia, a uva-do-japão floresce no Brasil de agosto a fevereiro e apresenta frutos maduros de março a outubro. Na Argentina, ela floresce de outubro a novembro e frutifica de maio até julho, em forma abundante, a partir de 4-5 anos após plantio (COZZO, 1960). No trabalho de Carminatti (1992), a frutificação inicia-se entre o $3^{\circ}$ e o $4^{\circ}$ ano no Rio Grande do Sul, e a queda das folhas dá-se desde os meses de abril a maio até fins de agosto.

Apresenta ramificação dicotômica, com copa globosa e ampla, com ramos pubescentes enquanto jovens. A casca apresenta-se com espessura total de até $15 \mathrm{~mm}$, sendo a casca externa lisa a levemente fissurada, pardo-escura a cinza-escura e casca interna esbranquiçada. O fruto é uma pequena cápsula globosa seca de $6 \mathrm{~mm}$ a $7 \mathrm{~mm}$ de diâmetro, contendo 2 a 4 sementes, preso ao pedúnculo cor de canela, que se torna espessado e carnoso ao madurar, com sabor doce e agradável. As sementes são de coloração alaranjada ou avermelhada quando recém-colhida e passando para marrom e preta com o tempo, mais ou menos circular, de $4 \mathrm{~mm}$ a $8 \mathrm{~mm}$ de diâmetro (CARVALHO, 1994).

O estudo da vegetação de porte arbóreo nas áreas urbanas constitui tema de averiguação bastante complexo relativamente novo no campo de conhecimento científico e que envolve a interdisciplinaridade de diversas áreas (ROSSETTI, TAVARES, PELLEGRINO, 2010). Este trabalho teve como objetivo o reconhecimento dos exemplares de Hovenia dulcis Thunberg,

Helayne Aparecida Maieves e Rosemary Hoffmann Ribani 
encontradas na arborização de ruas, parques, bairros da cidade de Curitiba - PR.

praças, escolas, faculdades e universidades em nove

\section{MATERIAL E MÉTODOS}

O município de Curitiba, composto por 75 bairros localiza-se na porção leste do Paraná (25²5’ S e 49¹6’ O), a aproximadamente $900 \mathrm{~m}$ de altitude. O clima da região é do tipo $\mathrm{Cfb}$, de acordo com a classificação de Köppen, subtropical úmido mesotérmico, sem estação seca definida. As temperaturas médias nos meses mais quentes (dezembro, janeiro e fevereiro) correspondem a 23 e $24{ }^{\circ} \mathrm{C}$ e, nos meses mais frios (junho, julho e agosto), a 12 e $13{ }^{\circ} \mathrm{C}$ (CAVIGLIONE et al., 2000; VIEIRA e BIONDI, 2008).

No levantamento, realizado entre os meses de janeiro e fevereiro de 2012, buscou-se identificar, localizar as coordenadas geográficas, além da coleta do material vegetativo (folhas, flores e ramos) para catalogação em exsicata, dos exemplares de Hovenia dulcis Thunberg, encontradas na arborização urbana do município, em bairros escolhidos aleatoriamente.

A identificação se deu a partir da descrição em literatura e imagens das folhas (Figura 1), das flores, frutos e sementes (Figura 2), que posteriormente foram confirmadas pelo Laboratório de Botânica da Universidade Federal do Paraná.

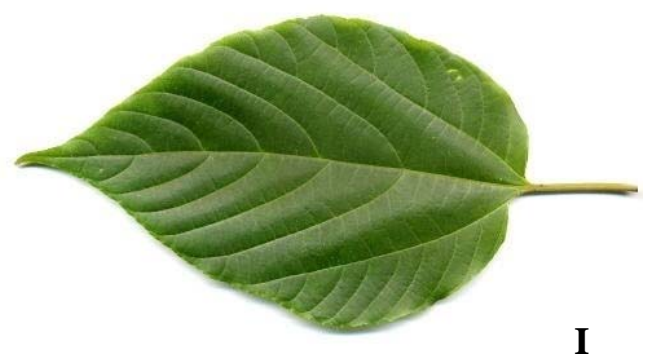

I

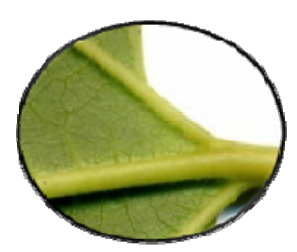

II

Figura 1. Anatomia da folha de Hovenia dulcis Thunberg, visão adaxial (I) e detalhe abaxial (II)

Fonte: KANON, 2003.

As coordenadas e referências, foram realizadas com auxílio de GPS, marca Garmin ${ }^{\circledR}$, modelo Nuvi 215W. A partir da coleta do material vegetativo, procederam as técnicas de secagem e posterior confecção de exsicata, para identificação em laboratório.

\section{RESULTADOS}

RECONHECIMENTO DE EXEMPLARES DE HOVENIA DULCIS THUNBERG... 
Foram encontrados ao todo 32 exemplares de Hovenia dulcis Thunberg, nos 9 bairros pesquisados (FIGURA 03), segundo descrito na tabela 1.
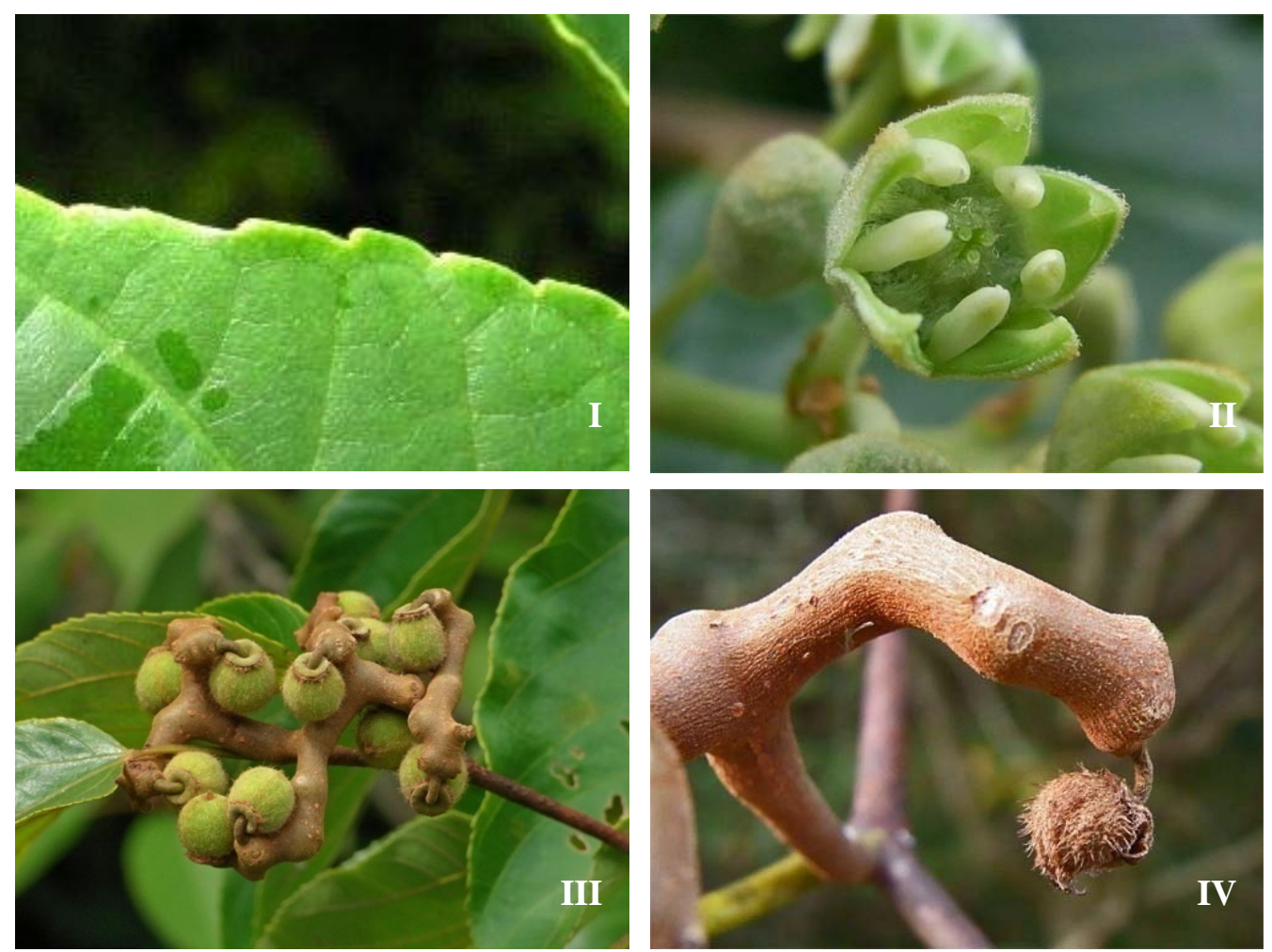

Figura 2. Anatomia da folha (I), flor (II), pseudofruto (pedúnculo frutífero) com sementes imaturas (III) e pseudofruto (pedúnculo frutífero) com sementes maduras (IV) de Hovenia dulcis Thunberg.

Fonte: KANON, 2003.

Tabela 1 Localização dos exemplares de Hovenia dulcis Thunberg, em diferentes bairros da cidade de Curitiba - PR

\begin{tabular}{|c|c|c|c|}
\hline $\mathrm{N}^{\circ}$ de exemplares & \multicolumn{2}{|c|}{ Coordenadas } & Bairros \\
\hline 08 & (S) $25^{\circ} 20^{\prime} 56^{\prime \prime}$ & (O) $49^{\circ} 13^{\prime} 57^{\prime \prime}$ & Jardim das Américas \\
\hline 03 & (S) $25^{\circ} 42^{\prime} 21^{\prime \prime}$ & 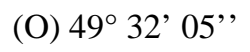 & Santo Inácio \\
\hline 03 & (S) $25^{\circ} 41^{\prime} 43^{\prime \prime}$ & (O) $49^{\circ} 25^{\prime} 61^{\prime \prime}$ & Alto da Glória \\
\hline 01 & (S) $25^{\circ} 42^{\prime} 22^{\prime \prime}$ & (O) $49^{\circ} 26^{\prime} 26^{\prime \prime}$ & Centro \\
\hline 06 & (S) $25^{\circ} 42^{\prime} 28^{\prime \prime}$ & (O) $49^{\circ} 25^{\prime} 29^{\prime \prime}$ & Alto da XV \\
\hline 02 & (S) $25^{\circ} 43^{\prime} 58^{\prime \prime}$ & (O) 49²5’08', & Cristo Rei \\
\hline 01 & (S) $25^{\circ} 44^{\prime} 35^{\prime \prime}$ & 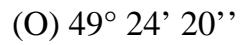 & Jardim Botânico \\
\hline 03 & (S) $25^{\circ} 46^{\prime} 19^{\prime \prime}$ & (O) $49^{\circ} 23^{\prime} 55^{\prime \prime}$ & Guabirotuba \\
\hline 05 & (S) $25^{\circ} 48^{\prime} 55^{\prime \prime}$ & (O) $49^{\circ} 25^{\prime} 01^{\prime \prime}$ & Hauer \\
\hline
\end{tabular}

HD: Hovenia dulcis - S: sul - O: oeste 


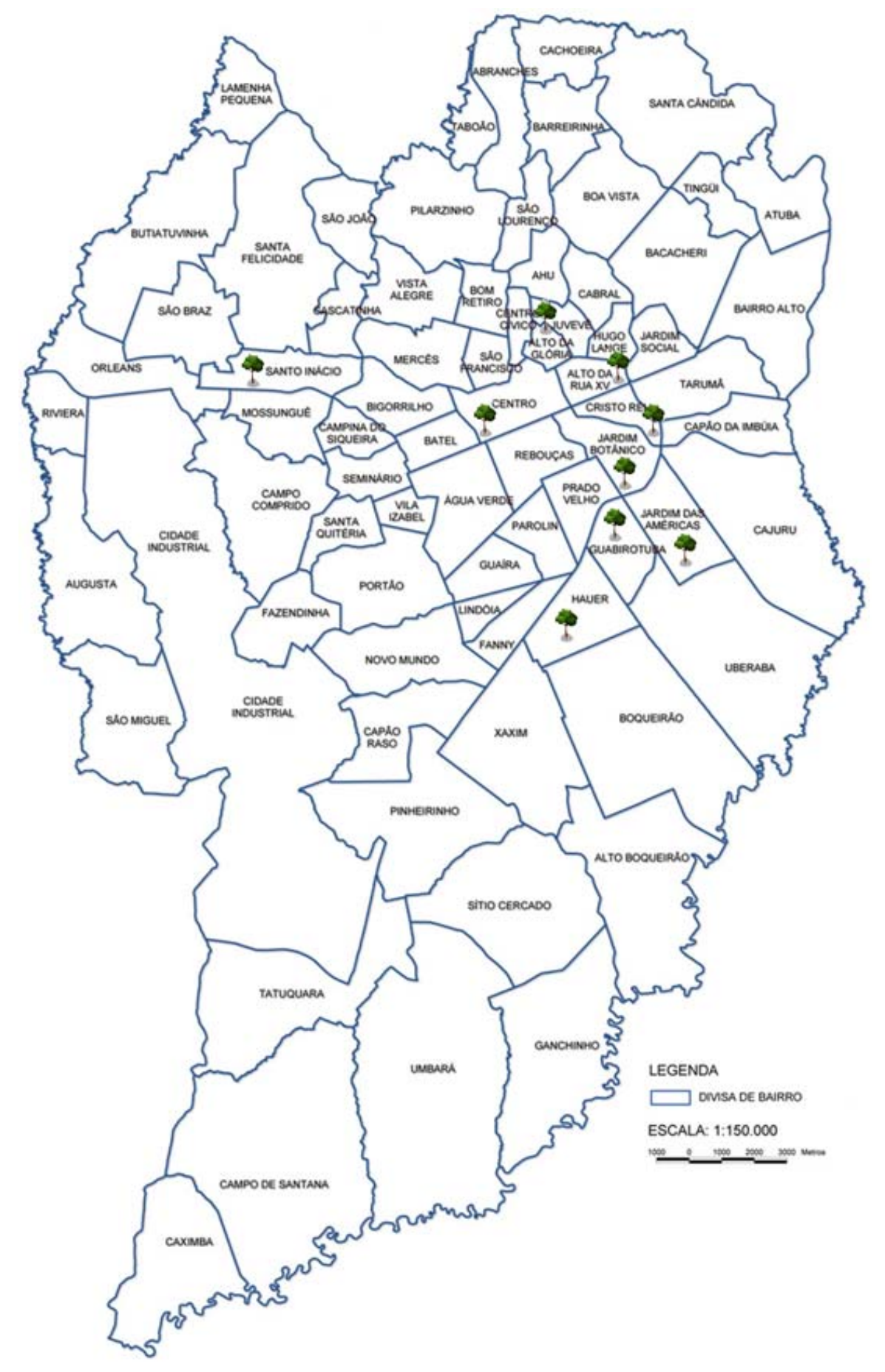

Figura 3. Mapa de Curitiba - PR e suas divisas, especificando os bairros onde foram encontrados exemplares de Hovenia dulcis, Thunberg.

Fonte: IPPUC, 2001.

RECONHECIMENTO DE EXEMPLARES DE HOVENIA DULCIS THUNBERG... 


\section{DISCUSSÃO}

No Campus III da Universidade Federal do Paraná, foram encontrados apenas 08 exemplares, conflitando com as 30 árvores de uva-do-japão que Leal, Pedroso-Macedo e Biondi (2009), encontraram num universo de 5034 plantas, correspondentes a 178 espécies, 134 gêneros e 67 famílias, numa área de 64 ha do Centro Politécnico. Uma das razões para explicar o decréscimo de árvores, são a ampliação da estrutura física, com novos prédios e estacionamentos dentro do Campus nos últimos três anos.

Observou-se que onde há exemplares de Hovenia dulcis Thunberg, ela se sobressaiu em relação à sua copa, sombreando espécies nativas, como os araçazeiros (Psidium cattleianum). Seu porte alcança a Araucaria angustifolia (Bertol.) Kuntze 1898, o pinheiro-do-paraná, que chega a medir 50 $\mathrm{m}$ de altura.

Também foram observados que sua floração ocorre entre os meses de agosto a dezembro, pois os materiais vegetativos que foram coletados predominaram-se folhas, ramos e frutos em fase de desenvolvimento inicial, nos meses de janeiro até a primeira quinzena de marçp. Assim conforme Cozzo (1960) descreveu nesta região, os frutos apresentam-se maduros, tão logo o início do outono, quando suas folhas começam a cair, ou seja, em meados do mês de março até o mês de junho.

No trabalho de Bobrowski (2011), que caracterizou a estrutura da arborização de ruas de Curitiba, comparando aos mesmos locais descritos por Milano em 1984, num total de 12 bairros, a saber, Água Verde, Alto da XV, Bacacheri, Bigorrilho, Boqueirão, Centro, Cristo Rei, Jardim Social, Mercês, Portão, Rebouças e Seminário.

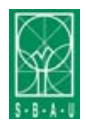

Milano (1984) encontrou 4.348 árvores, distribuídos pelos 12 bairros pesquisados, e encontrou apenas um exemplar de Hovenia dulcis Thunberg. Após 27 anos, Bobrowski (2011), encontrou um universo de 4.360 árvores, sendo 11 de uva-do-japão.

No trabalho de Carvalho, Nuci e Valaski (2010), onde foi realizado um inventário das árvores presentes na arborização de calçadas da porção central do bairro Santa Felicidade, região norte de Curitiba, não foi encontrada nenhuma uva-dojapão. Num total de 1806 árvores, os autores relataram que destas, 1094 não são consideradas frutíferas, ou seja, não produzem frutos comestíveis para consumo humano, 433 não foram identificadas e 279 são consideradas frutíferas, dentre elas muitas espécies nativas como a pitangueira (Eugenia uniflora), o araçazeiro (Psidium cattleianum), a guabirobeira (Campomanesia xanthocarpa) e o butiazeiro (Butia capitata).

Espécies como a Hovenia dulcis Thunberg e Melia azedarach têm grande facilidade de dispersão através de florestas ciliares sendo que em alguns locais no Paraná já constituem o estrato predominante de florestas aluviais, tomando agressivamente o espaço de diversas espécies nativas (SAMPAIO et al., 2011).

De acordo com Biondi e Macedo (2008), Curitiba é uma cidade cuja maior parte da população descende de imigrantes italianos, poloneses, alemães, ucranianos, japoneses, sírios e libaneses, característica que contribui para a utilização de espécies vegetais exóticas. Outro fator que deve ser considerado é a falta de pesquisas voltadas à produção de espécies nativas, as quais são, muitas vezes, pouco difundidas nos programas de Helayne Aparecida Maieves e Rosemary Hoffmann Ribani 
melhoramento e domesticação. Essa falta de pesquisas gera a falta de conhecimento por toda a cadeia produtiva, especialmente a de plantas ornamentais. Assim, produtores, profissionais (jardineiros, paisagistas, etc.) e consumidores finais carecem de informação a respeito da diversidade vegetal local e acabam por utilizar espécies exóticas.

As espécies exóticas são aquelas que, independentemente de serem ornamentais ou não, têm origem em outro território (BIONDI, 2004). Espécies exóticas invasoras são aquelas que ocorrem numa área fora de seu limite natural historicamente conhecido, como resultado de dispersão acidental ou intencional por atividades humanas. Atualmente, as espécies exóticas invasoras são reconhecidas como a segunda causa mundial para a perda de diversidade biológica, perdendo apenas para a destruição de hábitats e a exploração humana direta. Essas espécies, quando introduzidas em outros ambientes, livres de inimigos naturais se adaptam e passam a reproduzir-se a ponto de ocupar o espaço de espécies nativas e produzir alterações nos processos ecológicos naturais, tendendo a se tornar dominantes após um período de tempo mais ou menos longo requerido para sua adaptação (ZILLER et al., 2004).

Um resultado negativo encontrado no trabalho de Biondi e Leal (2008), que buscaram identificar as espécies produzidas no Horto Municipal da Barreirinha, em Curitiba-PR, foi que neste viveiro há produção de mudas de espécies consideradas invasoras. Um percentual de 6,67\% das espécies produzidas no Horto são invasoras no Brasil e no Paraná, como a Hovenia dulcis Thunberg, além das espécies: Acacia longifolia, Acacia mearnsii, Acacia podalyriaefolia, Casuarina equisetifolia,
Coffea arabica, Eryobotrya japonica, Morus nigra, Psidium guajava, Schizolobium parahyba e Spartium junceum.

De acordo com a Portaria $\mathrm{n}^{\circ}$ 95, de 22 de maio de 2007 (PARANÁ, 2007), que reconhece a Lista Oficial de Espécies Exóticas Invasoras para o Estado do Paraná, os imóveis públicos nos quais for constatada a presença de espécies exóticas invasoras, devem, obrigatoriamente, proceder à sua erradicação ou controle, para evitar a contaminação biológica. Assim, estas espécies não deverão ser mais produzidas neste viveiro. Para Mack et al. (2000) e Burt et al. (2007), a prevenção de iniciais introduções, ao invés de subsequente controle ou erradicação, é a forma mais efetiva de evitar gastos associados com combate de plantas invasoras. Ziller e Zalba (2007) afirmam que entre as ações para evitar e resolver problemas relacionados a espécies invasoras está o não cultivo de plantas ornamentais exóticas invasoras e a não utilização de espécies exóticas em projetos de restauração ambiental.

As espécies exóticas invasoras são consideradas a segunda maior causa de extinção de espécies no planeta, afetando diretamente a biodiversidade, a economia e a saúde humana (BRAND, 2006). Reconhecendo a importância do problema causado pelas invasões biológicas, a Convenção sobre a Diversidade Biológica - CDB - estabelece em seu Artigo $8^{\circ}$ que cada parte contratante deve, na medida do possível e conforme o caso, impedir a introdução, controlar ou erradicar as espécies exóticas invasoras que ameaçam ecossistemas, habitats e espécies nativas (MMA, 2006).

A prefeitura da cidade de Curitiba vem fazendo a retirada das espécies consideradas invasoras em parques do município, como foi o caso do Passeio Público, com cerca de 1.100 árvores, doze espécies 
são invasoras. Conforme a Secretaria Municipal do Meio Ambiente do município, para cada exótica invasora retirada, são plantadas cinco árvores nativas. (JUSBRASIL, 2010).

Segundo Bobrowski (2011) a espécie Hovenia dulcis Thunberg, não fazia parte de plantios planejados e executados pela Prefeitura de Curitiba, a partir de dados de 1984. Assim, tais constatações associam-se às afirmações de Biondi e Macedo (2008) de que a maioria das espécies invasoras presentes nas cidades são introduzidas pela população ou pelos órgãos públicos e privados.

As árvores ao longo das ruas, próximas às casas e em parques apresentam uma significância particular devido à interação mais intensa com as pessoas, pois influenciam positivamente o sentimento, as atitudes, o humor e o comportamento delas; também podem apresentar valores diferenciados conforme seu tamanho e sua localização (DWYER, 1995).

Apesar de serem consideradas invasoras, por não ser nativa da área em estudo, a uva-do-japão, apresenta uma madeira apreciável, como descrito por Carvalho (1994), sendo cultivado na região de Caxias do Sul - RS, porém efeitos alelopáticos em sementes de alface foram relatados por Rice, 1984 apud Wandscheer et al., 2011. Suas folhas possuem substâncias como as saponinas triterpênicas, muito estudadas na área de farmacologia, por possuírem atividade antiinflamatória (MALAQUIAS et al., 2000). Seus frutos são doces quando maduros e são alvo de estudos sobre seus teores de substâncias anticancerígenas, porém segundo Soares (1998) desaconselha à utilização de árvores que possuam frutos comestíveis como arborização de ruas, principalmente quando as mesmas sujam a pavimentação. Espécies frutíferas em arborização urbana é alvo de vandalismo, além de serem consideradas disseminadoras de doenças, por servirem de alimento para vetores, tais como moscas, ratos e barata (MILANO, 1996). O uso como alimento para animais, principalmente para ruminantes, não é indicado, pois no trabalho de Colodel et al. (1998), a doença Polioencefalomalacia (PEM) foi diagnosticada em caprinos que ingeriram, durante cinco dias Hovenia dulcis Thunberg.

\section{CONCLUSÃO}

A espécie Hovenia dulcis Thunberg, apresenta-se como uma espécie exótica, bem adaptada as condições climáticas e de solos na região sul. Apropriada para cultivos para fins de exploração de sua madeira, a uva-do-japão não se comporta bem como uma espécie para urbanização urbana, pois se tornou invasora, prejudicando a flora de espécies nativas.
A cidade de Curitiba - PR apresenta muitos exemplares de Hovenia dulcis Thunberg, em diversos bairros, localizados principalmente nas ruas e avenidas, escolas, faculdades e universidades. Já foram cultivadas em parques, porém hoje, a troca desta espécie por nativas é corrente, num processo de restaurar a flora nativa e erradicar espécies invasoras.

Helayne Aparecida Maieves e Rosemary Hoffmann Ribani 
BIONDI, D. Plantas invasoras na arborização urbana e paisagismo. In: PEDROSA MACEDO, J. H.; BREDOW, E. A. (Eds.). Princípios e rudimentos do controle biológico de plantas: coletânea. Curitiba: [s.n.]. p. 3-9, 2004.

BIONDI, D.; LEAL, L. Caracterização das plantas produzidas no Horto Municipal da Barreirinha - Curitiba / PR. Rev. SBAU, Piracicaba, v.3, n.2, p. 20-36, jun. 2008.

BIONDI, D.; MACEDO, J. H. P. Plantas invasoras encontradas na área urbana de Curitiba (PR). Floresta, 38(1): 129-144, 2008.

BRAND, K. et al. América do Sul invadida. A crescente ameaça das espécies exóticas invasoras. Programa Global de Espécies Invasoras (GISP), 2006.

BURT, J. W.; MUIR, A. A.; PIOVIA-SCOTT, J.; VEBLEN, K. E.; CHANG, A. L.; GROSSMANN, J. D., WEISKEL, H. W. Preventing horticultural introductions of invasive plants: potential efficacy of voluntaries initiatives. Biol. Invasions, v. 9, p. 909-923, 2007.

CARMINATTI, A. F. Contribuição ao estudo da germinação da espécie Hovenia dulcis Thun. (uva-do-japão). In: CONGRESSO FLORESTAL ESTADUAL, 7., 1992, Nova Prata. Anais. Santa Maria: Universidade Federal de Santa Maria. v.2, p.861- 881, 1992.

CARVALHO, P. E. R. Ecologia, silvicultura e usos da uva-do-japão (Hovenia dulcis Thunberg). Colombo: EMBRAPA Florestas, 1994. p. 24-65 (Circular Técnica EMBRAPA).

CARVALHO, J. A.; NUCI, J. C; VALASKI, S. Inventário das árvores presentes na arborização de calçadas da porção central do bairro Santa Felicidade - Curitiba - PR. REVSBAU, Piracicaba - SP, v.5, n.1, p.126-143, 2010.

CAVIGLIONE; J. H.; KIIHL, L. R. B.; CARAMORI, P. H.; OLIVEIRA, D. Cartas climáticas do Paraná. Londrina: IAPAR, 2000.

Colodel E. M., Loretti A. P., Cruz C. E. F., Driemeier D. Polioencefalomalacia em caprinos associada à ingestão de Hovenia dulcis (“Uva-do-Japão”). Boletim 18, Laboratório Regional de Diagnóstico, Pelotas, p.35-42, 1998.

COZZO, D. Resultados de Ias plantaciones florestais com Hovenia dulcis en Ia region Argentina subtropical y húmeda de Missiones. Revista Florestal Argentina, v.4, n.4, p.107-117, 1960.

DWYER, J. F. The significance of trees and their management in built environments. In: WATSON, G. W.; NEELY, D. (Ed.). Trees and Building sites: Proceedings of na International Workshop on Trees and Buildings. Savoy, IL : ISA, 1995. p.3-11.

JUSBRASIL. Árvores do Passeio Público ganham placas com nome e sobrenome, 23 de Abril de 2010. Disponível em: <http://prefcuritiba.jusbrasil.com.br/politica/47450 88/arvores-do-passeio-publico-ganhamplacas-com-nome-e-sobrenome>. Acesso em 03 de janeiro de 2011.

LEAL, L.; PEDROSA-MACEDO, J. H.; BIONDI, D. Censo da Arborização do Campus III - Centro Politécnico da Universidade Federal do Paraná. Scientia Agraria, Vol. 10, n. 6, 2009.

MALAQUIAS, F. S.; CARVALHO, T. M.; OLIVEIRA, E. F.; PELLICCIONE, V. L. B; GIANFALDONI, M. G.; FIGUEIREDO, MR; HENRIQUES M. G. M. O; ROSAS, E. C; VIANA, V. R. C.; ALBARELLO, N.; FIGUEIREDO, S. F. L. Atividade antiinflamatória de Cleome spinosa e Hovenia dulcis cultivadas in situ e in vitro. In: Resumos da XXII Reunião Anual sobre Evolução, Sistemática e Ecologia Micromoleculares. Rio de Janeiro, UFF; p-09, 2000. 
MACK, R. N.; SIMBERLOFF, D.; LONSDALE, W. M.; EVANS, H.; CLOUT, M.; BAZZAA, F. A. Biotic invasions: causes, epidemiology, global consequences and control. Ecol. Appl., n. 10, p. 689-710, 2000.

MILANO, M. S. Avaliação e análise da arborização de ruas de Curitiba-PR. 130f. Dissertação (Mestrado em Engenharia Florestal) - Setor de Ciências Agrárias, Universidade Federal do Paraná, Curitiba, 1984.

MILANO, M. S. Arborização Urbana no Brasil: Mitos e Realidade. In: III Congresso Brasileiro de Arborização Urbana, 11, 1996, Salvador. Anais..., 1996. p.1-6.

MMA - MINISTÉRIO DO MEIO AMBIENTE. Espécies Exóticas Invasoras: Situação Brasileira. Brasília: Ministério do Meio Ambiente, Secretaria de Biodiversidade e Florestas, 2006.

PARANÁ. Portaria IAP n 074, de 19 de abril de 2007. Reconhece a Lista Oficial de Espécies Exóticas Invasoras para o Estado do Paraná, estabelece normas de controle e dá outras providências.

RIGATTO, P. A.; PEREIRA, J. C. D.; MATTOS, P. P.; SCHAITZA, E. G. Características Físicas, Químicas e Anatômicas da Madeira de Hovenia dulcis. Comunicado Técnico - Embrapa Florestas - Colombo - PR, novembro - 2001.

ROssetTI, A. I. N.; TAVARES, A. R.; PELLEGRINO, P. R. M. Inventário arbóreo em dois bairros paulistanos, Jardim da Saúde e Vila Vera, localizados na subprefeitura de Ipiranga. Rev. Árvore. vol. 34, n.5, pp. 889-898, 2010.

SAMPAIO, A. C. F; ECKER, E. A.; MARANGONI, C. J. M.; FIORESE, L. M. R.; SORDI, E. A. Espécies Exóticas invasoras na arborização de vias públicas de três bairros de Campo Mourão - PR. Campo Digit@l, Vol. 6, No 1, 2011.

SELLE, G. L.; DIMAS FLEIG, F.; VUADEN, E.; JACQUES DE ALBERNARD, L. A.; MUÑOZ BRAZ, E. Índices de Sítios para Hovenia dulcis Thunberg na região central do estado do Rio Grande do Sul, Brasil. Ciência Florestal, Vol. 19, N. 4, out-dez, 2009, pp. 407-423.

SOARES, M. P. Verdes urbanos e rurais: orientação para a arborização de cidades e

sítios campesinos. Porto Alegre: Cinco Continentes.1998. 242p.

VIEIRA, C. H. S. D.; BIONDI, D. Análise da dinâmica da cobertura vegetal de Curitiba, PR (de 1986 a 2004), utlizando imagens Landsat TM. Rev. Árvore. 2 vol.32, n.3, pp. 479-487, 2008.

ZILLER, S. R.; ZALBA, S. Propostas de ação para prevenção e controle de espécies exóticas invasoras. Natureza e Conservação, Curitiba, v. 5, n. 2, p. 8-15, 2007.

ZILLER, S. R.; ZENNI, R. D.; NETO, J. G. Invasões biológicas: introdução, impactos e espécies invasoras no Brasil. In: Pedrosa-Macedo, J. H.; Bredow, E. A. (Eds.). Princípios e rudimentos do controle biológico de plantas: coletânea. Curitiba: [s.n.]. p. 17-41, 2004.

WANDSChEER, A. C. D.; BORELlA, J.; BONATTI, L. C.; PASTORINI, L. H. Atividade alelopática de folhas e pseudofrutos de Hovenia dulcis Thunb. (Rhamnaceae) sobre a germinação de Lactuca sativa L. (Asteraceae). Acta Bot. Bras. 2011. 